\title{
Functional conservation of the meiotic genes SDS and $R C K$ in male meiosis in the monocot rice
}

\author{
Ling Chang ${ }^{1}$, Hong $\mathrm{Ma}^{2,3}$, Hong-Wei Xue ${ }^{1}$ \\ ${ }^{I}$ National Key Laboratory of Plant Molecular Genetics, Institute of Plant Physiology and Ecology, Shanghai Institutes for Biologi- \\ cal Sciences, Chinese Academy of Sciences, 300 Fenglin Road, Shanghai 200032, China; ${ }^{2}$ Department of Biology and the Huck \\ Institutes of the Life Sciences, Pennsylvania State University, University Park, PA 16802, USA; ' School of Life Sciences, Fudan \\ University, Shanghai 200433, China
}

The Arabidopsis SDS (SOLO DANCERS) and RCK (ROCK-N-ROLLERS) genes are important for male meiosis, but it is still unknown whether they represent conserved functions in plants. We have performed phylogenetic analyses of $S D S$ and $R C K$ and their respective homologs, and identified their putative orthologs in poplar and rice. Quantitative real-time RT-PCR analysis indicated that rice $S D S$ and $R C K$ are expressed preferentially in young flowers, and transgenic RNAi rice lines with reduced expression of these genes exhibited normal vegetative development, but showed significantly reduced fertility with partially sterile flowers and defective pollens. SDS deficiency also caused a decrease in pollen amounts. Further cytological examination of male meiocytes revealed that the SDS deficiency led to defects in homolog interaction and bivalent formation in meiotic prophase $\mathrm{I}$, and $\mathrm{RCK}$ deficiency resulted in defective meiotic crossover formation. These results indicate that rice $S D S$ and $R C K$ genes have similar functions to their Arabidopsis orthologs. Because rice and Arabidopsis, respectively, are members of monocots and eudicots, two largest groups of flowering plants, our results suggest that the functions of $S D S$ and $R C K$ are likely conserved in flowering plants.

Keywords: rice, male meiosis, $S D S, R C K$, functional conservation

Cell Research (2009) 19:768-782. doi: 10.1038/cr.2009.52; published online 5 May 2009

\section{Introduction}

Meiosis is a reductional cell division for eukaryotic sexual reproduction and generates cells that contain half of the genetic materials from the parental cells and develop into germ cells. This process involves one round of DNA replication and two rounds of nuclear division, that is, meiosis I and meiosis II. Meiosis I has a prolonged and highly complex prophase I, which has five substages with characteristic chromosomal properties: leptotene, zygotene, pachytene, diplotene and diakinesis [1-5]. Previous studies showed that the chromosomes condense during the leptotene stage and form thin thread-like structures, then the homologs undergo pairing, synapsis

Correspondence: Hong-Wei Xue

Tel: +86-21-54924059; Fax: +86-21-54924060

E-mail: hwxue@sibs.ac.cn

Received 31 July 2008; revised 24 September 2008; accepted 10

November 2008; published online 5 May 2009 and recombination from zygotene to pachytene. The homologs undergo desynapsis in diplotene, but remain associated and form highly condensed bivalents at the diakinesis stage.

Homolog paring, synapsis and recombination have very close relationships, as all three processes involve the homologs and occur during overlapping periods. Homolog pairing initiates at the leptotene-zygotene transition and progresses through zygotene [6]. Homologs begin to synapse in zygotene and form synaptonemal complex (SC) at the pachytene stage. Meiotic recombination begins in late leptotene or zygotene and is completed in late pachytene. However, the nature of relationship between synapsis and recombination of homologs may differ between different organisms. In yeast, mouse and Arabidopsis thaliana, SC formation is dependent on the initiation of recombination by double-stranded DNA breaks (DSBs) [7-11]. In Caenorhabditis elegans and Drosophila, homolog synapsis occurs earlier than recombination, and SC formation does not require DSBs, but 
the initiation of recombination does $[12,13]$.

To ensure the proper meiosis, timing and order of various meiotic events are finely controlled. Numerous studies have demonstrated that cyclins and cyclin-dependent protein kinases $(\mathrm{CDKs})$ play pivotal roles in controlling major phases of the cell cycle [14-16]. In yeast, besides controlling mitotic cell cycle, cyclins and CDKs also function in regulating meiosis $[17,18]$. In mouse, the cyclin A1 is required for normal meiosis from pachytene to diplotene [19]. In Arabidopsis, a novel meiosisspecific cyclin-like protein named SDS (SOLO DANCERS) is required in homolog interaction during meiotic prophase I [20]. The $S D S$ gene is expressed in male and female meiotic cells, and $s d s$ mutant meiocytes are abnormal in synapsis, recombination and bivalent formation, resulting in abnormal chromosome distribution and defective meiotic products.

A widely accepted model for recombination, the double-stranded break repair model [21], was proposed based on the genetic and molecular studies in yeast and animal cells. According to the model, recombination is initiated by a DSB in one of the two participating molecules. Then some DSBs are converted to double-Holliday junctions, leading to the formation of recombination crossovers, whereas others are repaired via the synthesisdependent strand annealing intermediate to form noncrossovers [22]. In budding yeast, SPO11 functions in the generation of DSBs [23, 24]. MSH4, MSH5 and MER3 are required for normal crossover formation [2528]. MER3 is a DNA helicase and is important for extension of the DNA heteroduplex in the second end capture (SEC) intermediate [29]. Recent studies showed that a MER3 homolog in Arabidopsis, named as either ROCK$N$-ROLLERS (RCK) or AtMER3, is also necessary for crossover formation [30,31]. $R C K$ is preferentially expressed in the developing anthers and T-DNA insertional $r c k$ knockout mutants show defects in homolog synapsis and crossover formation; consequently, rck late prophase I meiocytes contain lesser than five bivalents and some univalents [30].

In many organisms, the occurance of a crossover alters the probability of a nearby second crossover formation that is expected from random distribution; this phenomenon is called interference [32-36]. Mutations in several genes, including MSH4, MSH5, MER3 and $R C K / A t M E R 3$, cause a dramatic reduction of crossovers (the residual crossovers is only about $10-15 \%$ of normal levels). Furthermore, the distributions of the remaining chiasmata in the mutants are not statistically different from predicted Poisson distributions, suggesting that the formation of the remaining crossovers is not sensitive to interference. However, the wild-type chiasma distribu- tion is dramatically different from the Poisson distribution, demonstrating that budding yeast and Arabidopsis both possess two genetically distinct pathways for crossover formation: a major interference-sensitive pathway dependent on MSH4/5 and MER3 proteins and a minor interference-insensitive pathway that is independent of these proteins [26, 37-40].

Although $S D S$ and $R C K$ have been shown to be important for meiosis in Arabidopsis, a member of the eudicot group of flowering plants, whether these genes represent conserved function in angiosperm is not known. The monocot rice is an excellent system for studying the molecular mechanism of meiosis [41]. In recent years, a few rice meiotic genes have been reported, including PAIR 1 [42], PAIR2 [43], OsDMC1 [44], OsRad21-4 [45] and OsRad21-3 [46]. To investigate the functional evolution of $S D S$ and $R C K$, we have identified the putative rice orthologs of $S D S$ and $R C K$, and studied their functions using RNAi-mediated reverse genetic approaches. Rice $S D S$ and $R C K$ were preferentially expressed in flowers, and suppression of either gene led to aberrant chromosome behaviors. Furthermore, crossover formation and bivalent formation were defective in $R C K$-deficient lines. The results indicate that rice $S D S$ is required for normal homolog interaction and $R C K$ is required for normal levels of crossover formation in meiosis I, revealing the conserved male meiosis mechanism in monocots and eudicots.

\section{Results}

Identification and phylogenetic analyses of putative orthologs of SDS and RCK from rice and other plants

Using the amino sequences of the Arabidopsis SDS (SOLO DANCERS) and RCK (ROCK-N-ROLLERS) proteins as queries, two rice genes, Os03g12414 and Os02g40450, encoding a putative cyclin and a putative RCK homolog, respectively, were identified by BLASTP search against the TIGR rice database. The predicted 5' and 3' UTRs from the Gramene database (http://www. gramene.org/Oryza_sativa_japonica/) were used to design primers for amplifying the rice $S D S$ and $R C K \mathrm{cD}$ NAs, respectively, by polymerase chain reaction (PCR). The amplified cDNAs were sequenced and comparison of the cDNA and genomic sequences reveals that the predicted $S D S$ exon/intron structure is correct, but that of $R C K$ is incorrect. The actual $R C K$ cDNA contains an 18 -bp exon between the theoretically predicted 22 nd and 23rd exons.

Similar to the Arabidopsis SDS protein, the predicted rice SDS protein contains two conserved cyclin domains: cyclin_N (spanning amino acids 239-375) and cyclin_C 
A

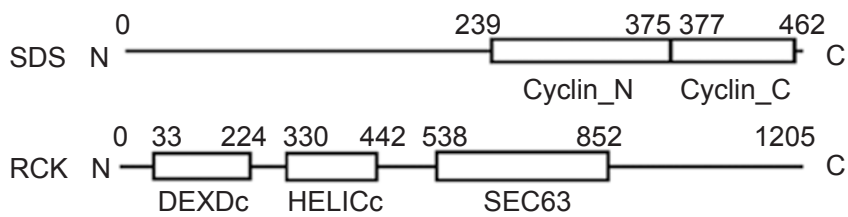
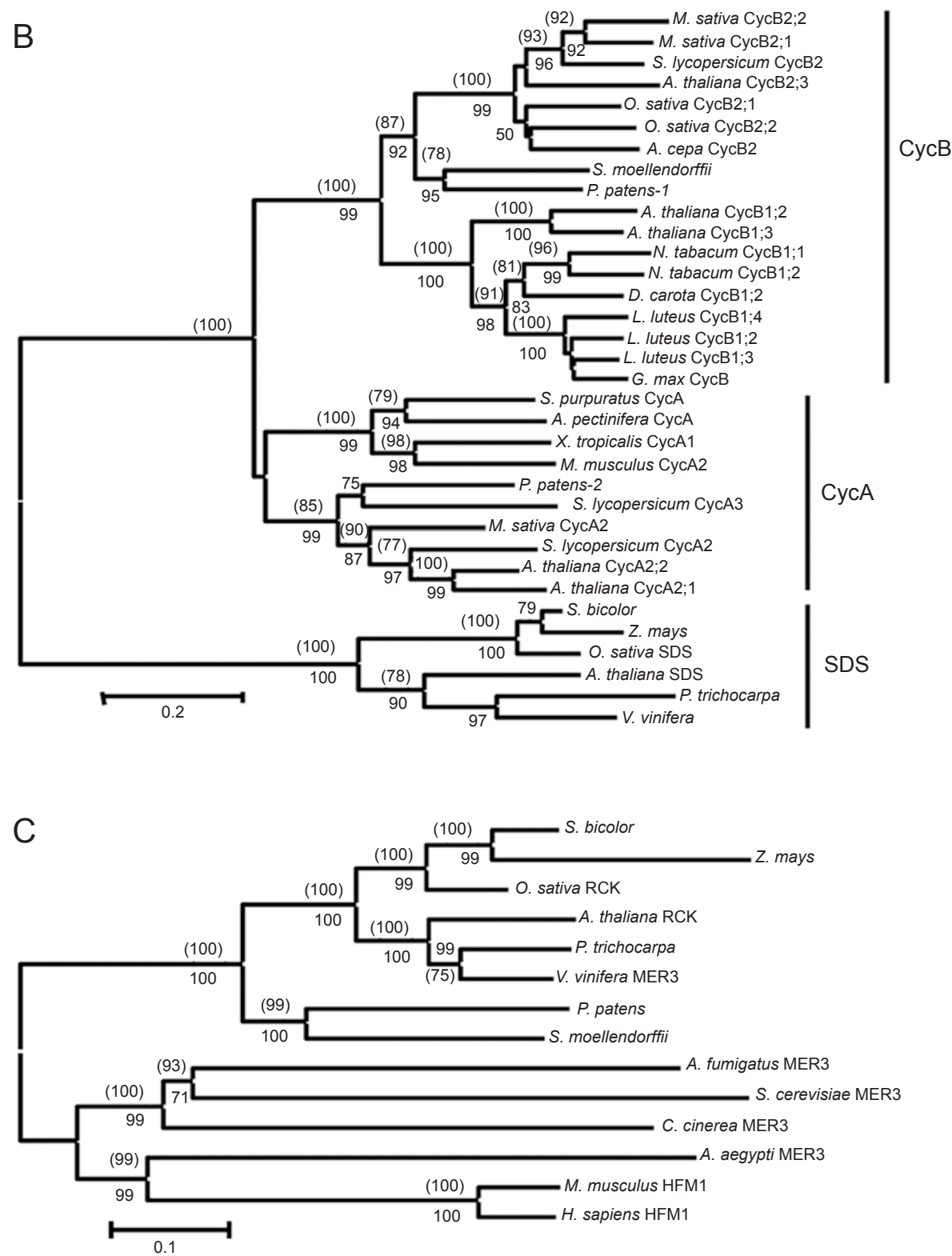

Figure 1 Structural organization and phylogenetic analyses of putative orthologs of rice SDS and RCK. (A) Structural organization of rice SDS and RCK. Positions of domains cyclin_N, cyclin_C, DEXDc, HELICc and SEC63 are indicated. (B) A neighbor-joining phylogenetic tree for SDS and its homologs in different organism, with bootstrap values higher than $50 \%$ shown for each clade. Bootstrap values in parentheses are from the tree generated using Maximum Likelihood method for the same sequence alignment. Except indicated, those homologous are with following accession numbers: S. moellendorffii (jgi|Selmo1|231762|fgenesh1 pm.); P. patens-1 (jgi|Phypa1_1|186054|estExt gwp); P. patens-2 (jgi|Phypa1_1|32594|gw1.1 47.60.); Z. mays (AC205249.1_FGP020); P. trichocarpa (eugene3.00100975) and V. vinifera (CAN78702). (C) A neighborjoining phylogenetic tree of $R C K$ and its homologs in different organisms. The bootstrap values in parentheses are given for Maximum Likelihood analysis. Except those indicated, those homologous are with following accession numbers: S. bicolor (jgi|Sorbi1|4812687|e gw1.4.145); Z. mays (AC188828.2_FG028); P. patens (jgi|Phypa1_1|122688|e gw1.41.1) and S. moellendorffii (Selmo1|423377|fgenesh2 pg.C sc). 
(amino acids 377-462) (Figure 1A, top panel), suggesting that rice SDS might have the activity to regulate CDKs. Sequence alignment showed that rice SDS has 49\% identity and 64\% similarity with SDS of Arabidopsis.

Sequence analysis showed that rice RCK has several conserved domains, including DEXDc (amino acid positions 33-224), HELICc (amino acids 330-442) and SEC63 (amino acid positions 538-852) (Figure 1A, bottom panel). DEXDc is found in members of a DEADlike superfamily involved in ATP-dependent RNA or DNA unwinding [47]. The HELICc domain is located at C-terminus of proteins that belong to a helicase superfamily, and is associated with DEXDc-, DEAD- and DEAH-box proteins [48]. The SEC63 domain was first found in the budding yeast SEC63 protein $[49,50]$. These domains are also found in the yeast MER3 and Arabidopsis RCK proteins and the presence of these domains suggests that RCK possibly has a helicase activity. The protein structures of rice SDS and RCK suggest that these two proteins may have conserved functions in rice. Further sequence comparison indicated that rice RCK has $73 \%$ identity and $87 \%$ similarity with Arabidopsis RCK.

To test whether $S D S$ and $R C K$ are conserved, we searched for sequences of additional putative $S D S$ and $R C K$ homologs and other related proteins. Putative $S D S$ homologs from poplar (Populus trichocarpa), grape (Vitis vinifera), maize (Zea mays) and sorghum (Sorghum bicolor) were identified, as well as representative genes for cyclin A and B. Alignment and phylogenetic analysis using neighbor-joining and maximum likelihood methods yielded trees with the same topology (Figure 1B). The six $S D S$ orthologs form a clade with high bootstrap support separate from the clades for cyclins A and B, suggesting that $S D S$ genes are derived from a single gene in the ancestor of flowering plants. This is consistent with the previous finding that the Arabidopsis and rice $S D S$ genes form a distinct clade among plant cyclin genes [51]. Because the cyclin A clade also contains animal genes, this further suggests that the $S D S$ clade was formed prior to the divergence of animal and plants. We have also retrieved sequences of putative $R C K$ homologs from several plants and representative non-plant species and constructed a phylogenetic tree (Figure 1C). The topology of the RCK is in agreement with the current understanding of the eukaryotic species relationship, indicating that the plant $R C K$ genes are likely orthologs among themselves.

\section{SDS and RCK are both preferentially expressed in flowers}

Sequence and phylogenetic analyses indicate that $S D S$ and $R C K$ are conserved in angiosperms, suggesting that they might have similar functions in flowering plants. To
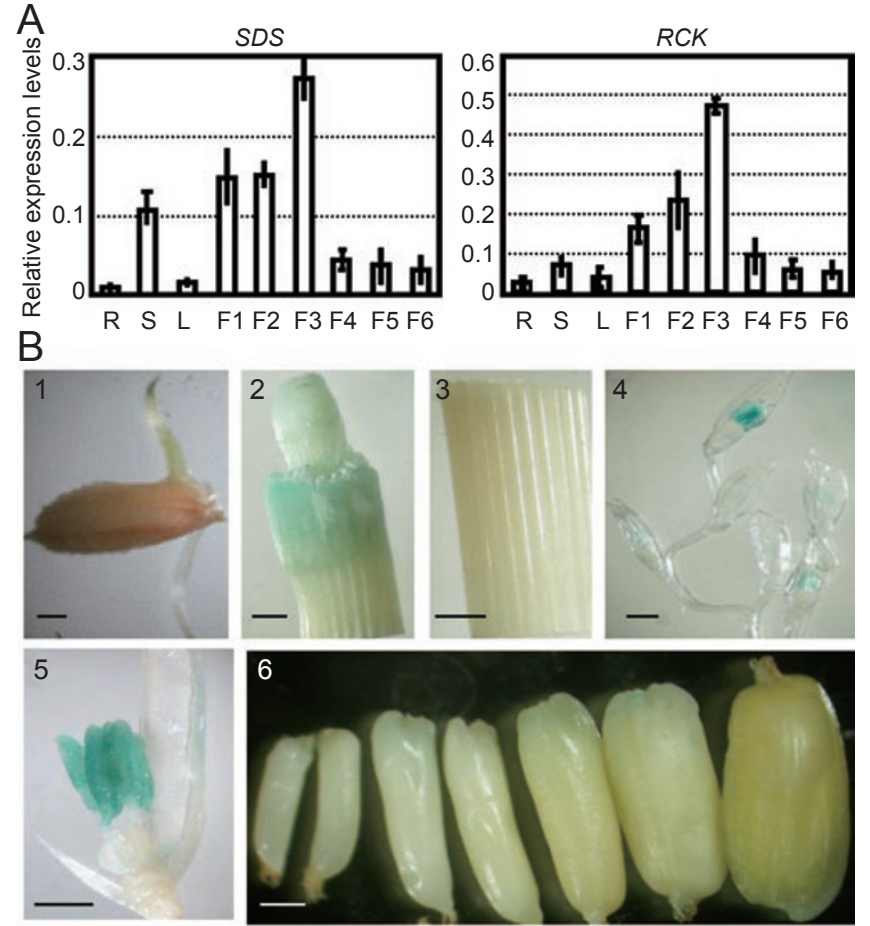

C
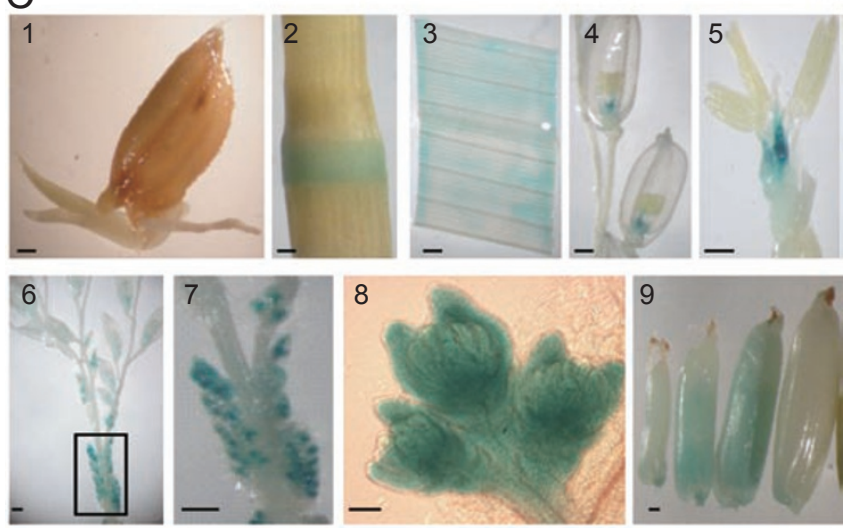

Figure 2 Expression of SDS and RCK. (A) Quantitative realtime PCR analysis reveals the expression of SDS (left panel) and $R C K$ (right panel) in various tissues and developing flowers, including root $(R)$, stem $(S)$, leaves $(L)$, stamen and carpel primordial formation (F1), pollen mother cell formation (F2), male meiosis (F3), uninucleate microspore (F4), bicellular pollen (F5) and tricellular pollen (F6). Relative mRNA levels were determined by normalizing the PCR threshold cycle number of each gene with that of the ACTIN1 reference gene. The results are presented as an average of three independent experiments. (B) Promoter-reporter gene fusion studies indicated that rice SDS is transcribed in the shoot apex of stem (2), panicle (4) and anther (5); and no expression is seen in 4-day seedling (1), mature leaf (3) and grains in different development stages (6). Bar $=1 \mathrm{~mm}$. (C) Histochemical analysis revealed that rice RCK is transcribed in stem node (2), mature leaf (3), flowers (4), flower with lemma and palea removed $(5)$, panicle $(6$, highlighted in 7,8$)$ and grains in different development stages (9). Bar=100 $\mu \mathrm{m}$ (8) and $1 \mathrm{~mm}(1-7,9)$. 
obtain clues about their functions, the expression of $S D S$ and $R C K$ in rice was examined by real-time quantitative RT-PCR using total RNAs extracted from vegetative and reproductive tissues. Floral stages were determined according to floral length and cytological observations, essentially as described previously [46]. Flowers with lengths of $\leq 0.8 \mathrm{~mm}$ were pooled as F1 (stamen and carpel primordial formation), flowers of $0.8-2 \mathrm{~mm}$ in length as F2 (pollen mother cell formation), those $2-4 \mathrm{~mm}$ long as F3 (male meiosis) and flowers of $4-6 \mathrm{~mm}$ in length as F4 (microspore formed). Flowers of $6 \mathrm{~mm}$ or longer, but before the heading stage and with anthers that were turning yellow were pooled as F5 (bicellular pollen). In the panicles (inflorescences) after heading, flowers close to anthesis were pooled as F6 (tricellular pollen stage). Quantitative real-time PCR analysis revealed that SDS was expressed preferentially in flowers and stems, with relatively weak expression in roots and leaves (Figure $2 \mathrm{~A}$, left panel). The level of $S D S$ transcript peaked at F1-F3 and decreased thereafter. Similarly, $R C K$ was expressed preferentially at F1-F3 as well (Figure 2A, right panel). These results suggested that both $S D S$ and $R C K$ were expressed at early stages of meiocyte development and during meiosis.

To investigate the expression patterns of $S D S$ and $R C K$ in detail, reporter gene fusion studies were performed with the $S D S$ or $R C K$ promoters and the GUS gene coding for $\beta$-glucuronidase (GUS). More than 20 independent transformants were obtained and analysis of the GUS activities in 10 transgenic lines revealed that $S D S$ was expressed in the shoot apex (Figure 2B, 2) and flowers (Figure 2B, 4), preferentially in anthers (Figure 2B, 5). On the other hand, $S D S$ expression was not detected in seedlings (Figure 2B 1), leaves (Figure 2B, 3) and grains at different developmental stages (Figure $2 \mathrm{~B}, 6$ ). $R C K$ was expressed in the node of stem (Figure 2C, 2), leaves (Figure 2C, 3), pistils of the mature flower (Figure 2C, 4, 5 ), panicles (Figure 2C,6) and panicles before meiosis stage (Figure 2C, 7), anthers and pistils of flowers before meiosis stage (Figure 2C, 8), and developing seeds (Figure $2 \mathrm{C}, 9)$.

Suppression of SDS results in defective pollen and reduced fertility

To characterize the in vivo function of $S D S$, we generated a gene-specific p35S SDS-RNAi construct using a 535-bp fragment of SDS cDNA (nt 430-964) that shares no similarity to any other sequences in the rice genome. A total of 30 independent transformants were identified by hygromycin resistance and the presence of the T-DNA in the rice genome was verified by PCR with primers localized to the spacer and the inserted cDNA sequences
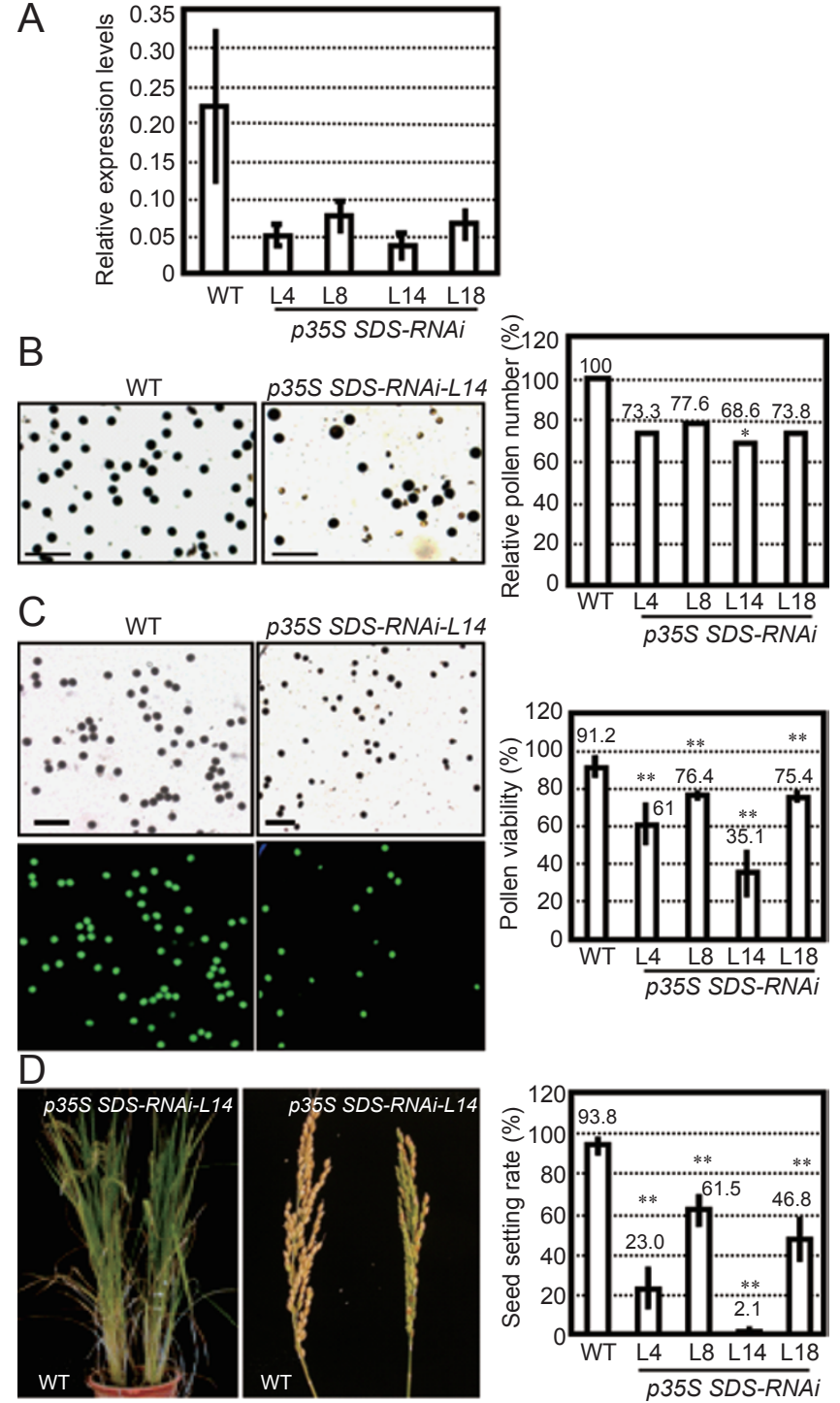

Figure 3 Suppressed expression of SDS resulted in reduced number and viability of pollen grains, and delayed seed setting rate. (A) Quantitative real-time PCR analysis of SDS mRNA levels in WT and independent transgenic lines $(L 4,8,14,18)$ by RNAi strategy. Total RNAs were extracted from flowers. mRNA levels are normalized relatively to the ACTIN1 level. Results are presented as an average of three independent experiments. (B) Viability of mature pollen grains in WT (left) and representative transgenic line (p35S SDS-RNAi-L14, middle), examined with iodium potassium iodide solution (left panel). The relative number of pollen grains is counted using a hematocyte arithmometer, with that of WT as control (right). Statistical analysis using one-tailed Student's $t$-test indicated the significant difference $\left({ }^{*} P<0.05\right)$. Bar $=$ $200 \mu \mathrm{m}$. (C) Pollen viability analysis of WT (left) and representative transgenic line $p 35 S$ SDS-RNAi-L14 (middle) with FDA staining. The relative viability is calculated and shown in percentage (right). Statistical analysis using one-tailed Student's $t$-test indicated the significant differences $(* * P<0.01)$. Bar $=200 \mu \mathrm{m}$. (D) Growth of plant (left), panicle (middle) and seed setting rate (right) of WT and transgenic line p35S SDS-RNAi-L14 (T1 generation). Statistical analysis using onetailed Student's $t$-test indicated the significant differences $(* * P<0.01)$. 
of vector p35S SDS-RNAi. Further analysis using realtime RT-PCR indicated that the $S D S$ expression was suppressed and lower than that in WT (Figure 3A). The $S D S$-RNAi lines developed normally during the vegetative stage, but seed production was severely disrupted as compared to WT plants. The mean seed setting rate (number of seeds as a percentage of the total number of flowers) at maturity of $p 35 S S D S-R N A i$ lines was greatly reduced $(23.0 \%, 61.5 \%, 2.1 \%$ and $46.8 \%$ of lines $L 4$, L8, L14 and L18, respectively), compared to that of WT (93.8\%) (Figure 3D), indicating that suppression of SDS in the RNAi lines results in a sterility phenotype.

As pollen development is important for the successful fertilization of sexual plants, the pollen development of rice SDS-RNAi lines (L4, L8, L14 and L18) in the T1 generation was compared with that of wild type. Mature anthers from the deficient lines did not differ obviously in morphology from those of wild type, but in an $\mathrm{I}_{2}-\mathrm{KI}$ staining assay, pollen grains from the wild type were round and had a uniform size, with a dark blue-black reaction (Figure 3B, left panel); in contrast, those of the $S D S$-RNAi lines were variable in size and shape (Figure $3 \mathrm{~B}$, middle panel). We also estimated the pollen amount per flower in these lines and found that the SDS-RNAi lines contained decreased amounts of pollen grains than wild type. Each flower of the SDS-RNAi lines L4, L8, L14, L18 had $73.3 \%, 77.6 \%, 68.6 \%$ and $73.8 \%$ of the wild-type amount of pollen grains on average, respectively (Figure 3B, right panel).

Further examination on the pollen viability using fluorescein diacetate (FDA) showed that the $S D S$-RNAi lines had significantly reduced pollen viability. Compared to $91.2 \%$ viability of WT pollen grains $(n=4889)$, those of SDS-RNAi lines had 61\% (L4, $n=2344$ ), 76.4\% (L8, $n=2667), 35.1 \%(\mathrm{~L} 14, n=2187)$ and $75.4 \%(\mathrm{~L} 18, n$ $=4188$ ) viable pollens, respectively (Figure 3C), consistent with the levels of suppression of $S D S$ expression, indicating that pollen viability is disrupted under $S D S$ suppression.

Suppression of $R C K$ results in reduced pollen viability and reduced fertility

The physiological function of rice $R C K$ was studied by a similar RNAi approach. Briefly, a rice $R C K$ RNAi vector (p35S $R C K$-RNAi) was constructed with a 514-bp cDNA fragment of $R C K$ (nt 2 409-2 922), which shares no similarity to other sequences in the rice genome. After transformation, 40 independent $\mathrm{T} 0$ transformants were obtained and most of them were confirmed to carry the T-DNA by PCR analysis. Further expression analysis on the 10 selected plants by quantitative real-time RT-PCR analysis confirmed the suppressed expression of endog- enous $R C K$ in several independent lines (L9, L12, L13 and L19), especially L12 (Figure 4A).

Similar to the $S D S$-RNAi lines, $R C K$-RNAi lines grew and developed normally during their vegetative stage,
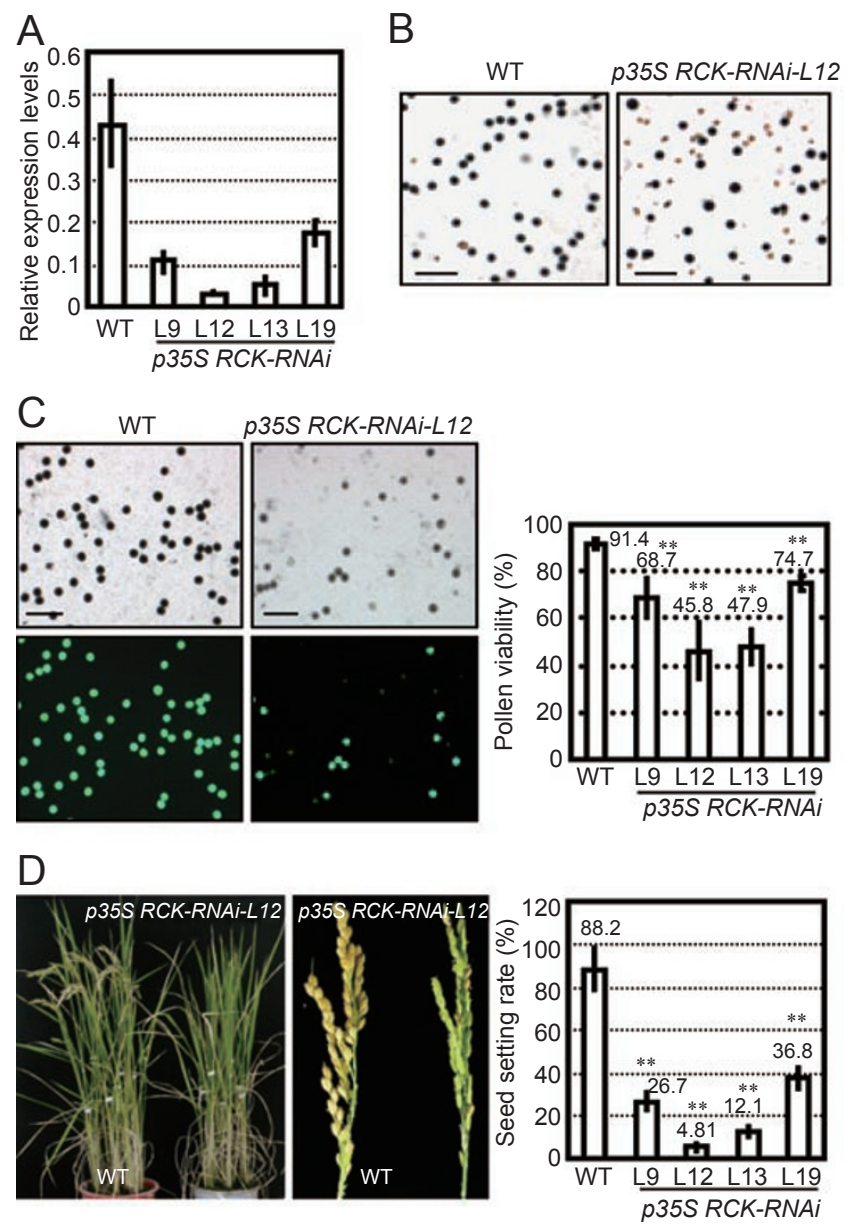

Figure 4 Suppressed expression of RCK resulted in an altered viability of pollen grains, and seed setting rate. (A) Quantitative real-time PCR analysis of RCK mRNA levels in WT and independent transgenic lines $(L 9,12,13,19)$ by RNAi strategy. Total RNAs were extracted from flowers. mRNA levels are normalized relatively to the ACTIN1 level. The results are presented as an average of three independent experiments. (B) Viability of mature pollen grains in WT (left) and representative transgenic line ( $p 35 S R C K-R N A i-L 12$, right), examined with iodium potassium iodide solution (left panel). Bar $=200 \mu \mathrm{m}$. (C) Pollen viability analysis of WT (left) and representative transgenic line p35S RCK-RNAi-L12 (middle) with FDA staining. The relative viability is calculated and shown in percentage (right). Statistical analysis using one-tailed Student's $t$-test indicated the significant differences $(* * P<0.01)$. Bar $=200 \mu \mathrm{m}$. (D) Growth of plant (left), panicle (middle) and seed setting rate (right) of WT and transgenic line p35S RCK-RNAi-L12 (T1 generation). Statistical analysis using one-tailed Student's $t$-test indicated the significant differences $(* * P<0.01)$. 
but seed production was severely disrupted. Calculation on the mean seed setting rate at seed maturity showed that, compared to WT plants (seed setting rate of $88.2 \%$ ), $R C K$ suppression resulted in the rate of $26.7 \%, 4.81 \%$, $12.1 \%$ and $36.8 \%$ (lines L9, L12, L13 and L19), respectively (Figure 4D), suggesting that knockdown of rice $R C K$ in RNAi lines is related to their sterility.

Observation on the pollen development and fertility showed that $R C K$ suppression did not result in altered morphology of mature anthers; however, in an $\mathrm{I}_{2}-\mathrm{KI}$ staining assay, pollen grains of the $R C K$-RNAi lines were variable in size and shape. Pollen grains from the WT were round and had a uniform size, with a dark blueblack staining (Figure 4B, left panel). In contrast, the number of pollen grains that were stained (viable) was greatly reduced in the $R C K$-RNAi lines and some grains were empty, shrunken and stained brown (Figure 4B, right panel). The pollen amount per flower was similar to that of WT, unlike the SDS-RNAi lines.

The FDA staining assay indicated that the pollen viability of the $R C K$-RNAi lines was significantly reduced. Compared to the $91.4 \%$ pollen viability of WT ( $n=2427)$, those of $R C K$-RNAi lines were only $68.7 \%$ (L9, $n=4$ $023), 45.8 \%(\mathrm{~L} 12, n=2057), 47.9 \%(\mathrm{~L} 13, n=4321)$ and $75.4 \%$ (L19, $n=3179$ ), respectively (Figure 4C), indicating that pollen viability was disrupted in $R C K$ RNAi lines.

\section{Suppression of SDS and RCK results in defective male meiosis I}

To evaluate whether the abnormal pollen development resulted from defective meiosis, we performed detailed cytological analysis of meiosis in male meiocytes from the SDS-RNAi (L4, L8, L14 and L18) and $R C K$-RNAi lines (L9, L12, L13 and L19). In WT, leptotene chromosomes appeared as thin threads that looped out of dense synizetic knots (Figure 5A). Homologous chromosomes began to associate side by side at zygotene (Figure 5B), and fully synapsed into thick threads at pachytene (Figure 5C). After synapsis was resolved at diplotene, except at chiasmata (Figure 5D), 12 bivalents became tightly condensed at diakinesis (Figure 5E) and were arranged at the equatorial plate at metaphase I (Figure 5F). At anaphase I, homologs separated (Figure 5G) and moved towards opposite poles of the spindle, forming two clusters at telophase I (Figure 5H).

In both $S D S$ and $R C K$ RNAi lines, chromosomal patterns similar to the WT leptotene through pachytene stages were observed (Figure 5I-5K, 5Q-5S). Although it was difficult to determine whether diplotenes in $S D S$ and $R C K$-deficient lines were normal (Figure $5 \mathrm{~L}$ and $5 \mathrm{~T}$ ), it was obvious that, by diakinesis, the homologs were not all attached in SDS-RNAi cells with 24 univalents
(Figure 5M). In RCK-RNAi cells, there were more than 12 distinguishable chromosomes, indicating the presence of univalents (Figure 5U). Furthermore, comparison of WT and transgenic meiocytes at late stages of meiosis I revealed additional defects, which may be a result of the defective prophase I (Figure $5 \mathrm{~N}-5 \mathrm{P}$ and $5 \mathrm{~V}-5 \mathrm{X}$ ). In $p 35 \mathrm{~S}$ $S D S-R N A i-L 14$ and $p 35 S$ RCK-RNAi-L12 cells at metaphase I, chromosomes often did not align at the equatorial plane (Figure $5 \mathrm{~N}$ and $5 \mathrm{~V}$ ); some of them were quite far from the equator (arrowheads in Figure $5 \mathrm{~N}$ and $5 \mathrm{~V}$ ). The anaphase I cells showed more than two clusters of chromosomes and several lagging chromosomes (arrowheads in Figure $5 \mathrm{O}$ and $5 \mathrm{~W}$ ). Then at telophase I, in $p 35 \mathrm{~S}$ $S D S-R N A i-L 14$ cells, some chromosomes still stayed at the center (arrowhead in Figure 5P) and chromosome bridges were observed (arrow in Figure 5P); in $p 35 S$ $R C K-R N A i-L 12$ cells, some chromosomes were delayed in moving to the poles (arrowheads in Figure 5X).

\section{Altered meiocyte distributions under SDS or RCK sup- pression}

Male meiosis in rice is slightly asynchronous and a population of male meiotic cells from a single flower can cover a few adjacent meiotic stages or substages of the long prophase I [41]. We noticed that $p 35 S S D S-R N A i$ and $p 35 S R C K-R N A i$ cells with chromosome images similar to that in Figure $5 \mathrm{~K}$ and $5 \mathrm{~S}$ (pachytene) were very infrequent, suggesting defects in synapsis. Then, we examined a large number of meiocytes from wild type, p35S SDS-RNAi-L14 and p35S RCK-RNAi-L12 to analyze the meiocyte distribution, similar to the examination of the Arabidopsis sds mutant [20]. The results showed that among the wild-type prophase I cells (1954 cells), nearly $31 \%$ were pachytene cells and relatively few were leptotene cells (Figure 6A). In contrast, the p35S SDSRNAi-L14 showed a dramatically different distribution of prophase I substages ( 2138 cells); the number of cells at the zygotene was much higher than normal, whereas cells at the pachytene were rare. The pattern of $p 35 \mathrm{~S}$ $S D S-R N A i-L 14$ distribution of prophase I stages supports the idea that synapsis is defective in $S D S$-deficient transgenic lines. Similarly, the distribution patterns of wildtype (3 232 cells) and $p 35 S R C K-R N A i-L 12$ (3 842 cells) meiosis I meiocytes indicate that $p 35 S R C K-R N A i-L 12$ also had increased frequencies of cells at the leptotene and zygotene stages, and reduced frequencies of cells at diakenesis, metaphase I, anaphase I and telophase I (Figure $6 \mathrm{~B})$.

Suppression of RCK results in decreased numbers of chiasmata and bivalents

The fact that some bivalents were observed in $R C K$ - 

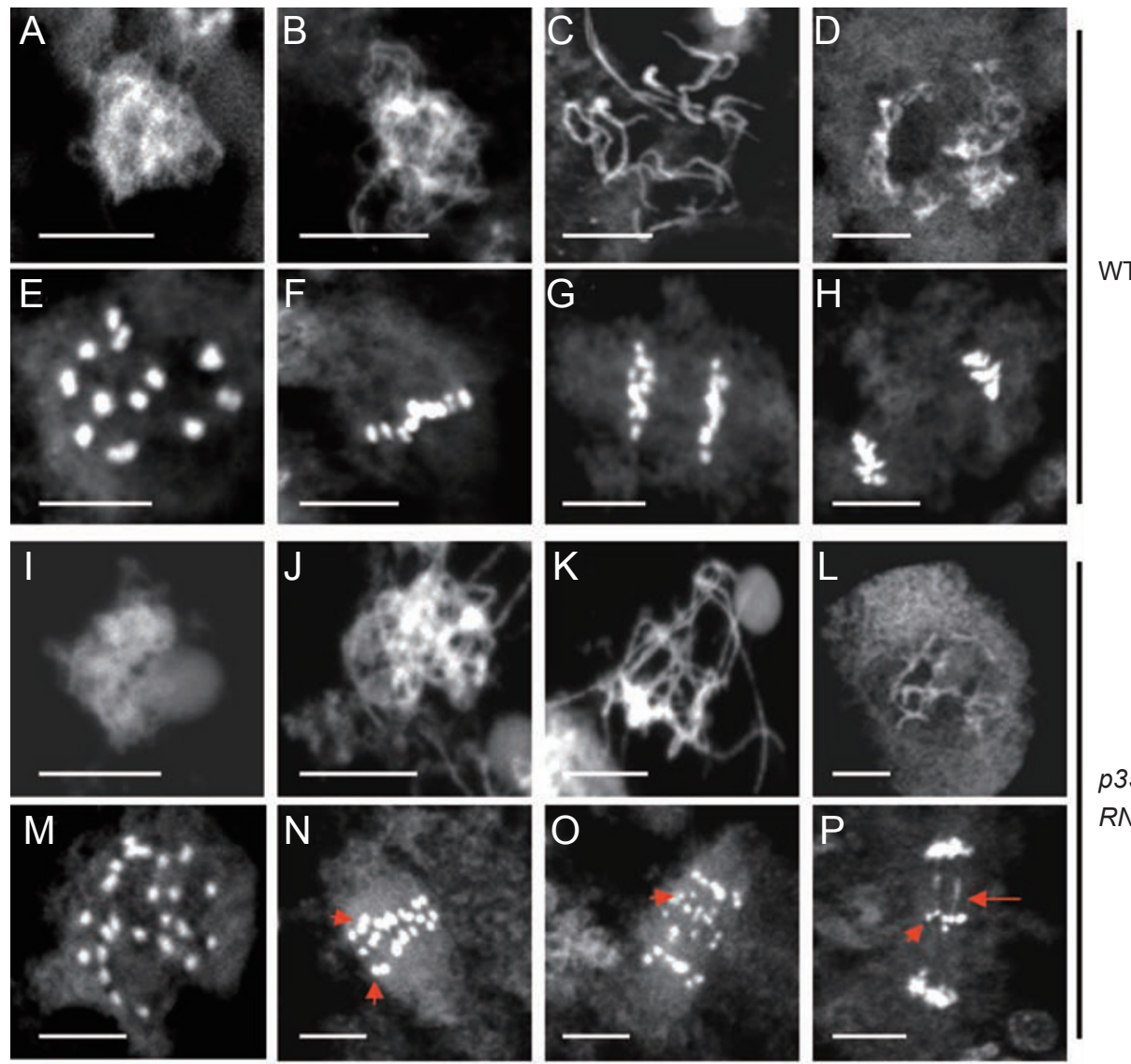

p35S SDS-
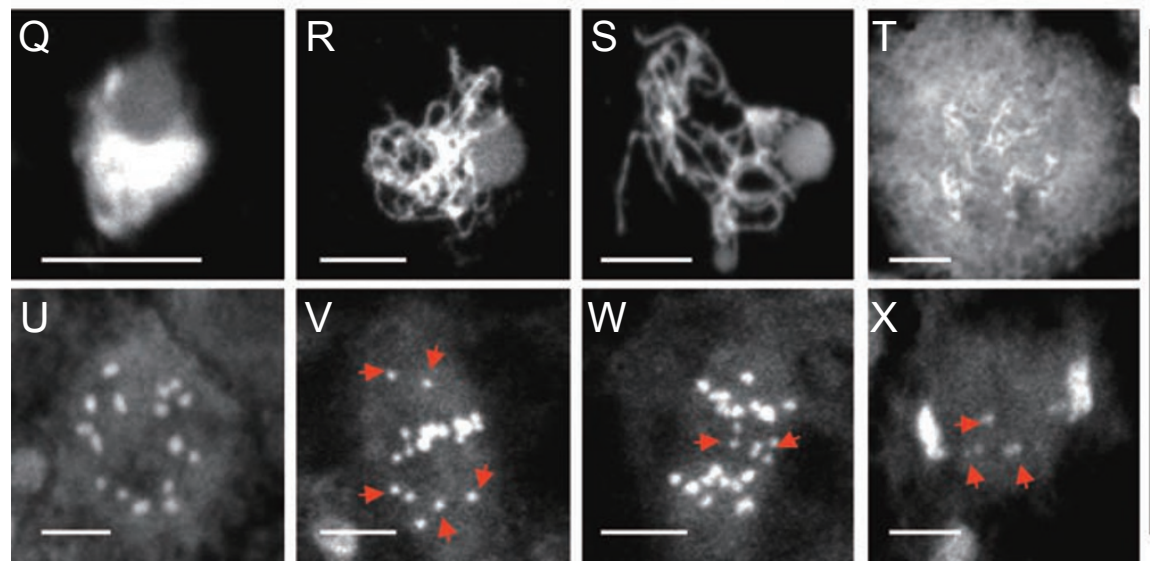

p35S RCK-

RNAi

Figure 5 Male meiosis I in WT and transgenic lines with suppressed expressions of SDS or RCK. The DAPI-stained chromosomes are shown. Prophase I at leptotene, zygotene, pachytene and diplotene, are similar in WT (A-D), p35S SDS-RNAi lines (I-L) and p35S RCK-RNAi lines (Q-T), while the diakenesis of prophase I, metaphase I, anaphase I and telophase I are significantly altered. At diakenesis of prophase I, 12 attached pairs of condensed homologs are formed in WT (E); while 24 stained bodies can be observed in p35S SDS-RNAi lines, indicating that the condensed homologs formed univalents (M); 16 stained bodies can be observed in p35S RCK-RNAi lines, indicating a mixture of bivalents and univalents (U). At metaphase I, the chromosomes align at the equator in WT (F); while those that did not align on equator in p35S SDS-RNAi lines (arrowheads, $\mathbf{N}$ ) or did not all align at the equator in p35S RCK-RNAi lines (arrowheads highlight that some chromosomes are away from the equator, V). At anaphase I, the homologs are separating in WT (G); while the chromosomes are elongated similarly to those at metaphase I in p35S SDS-RNAi lines, suggesting that they might be pulled by the spindle as normal homologs are at anaphase I (arrowhead highlights the lagging chromosomes, 0); or some chromosomes are with delayed elongation (arrowheads) in p35S RCK-RNAi lines (W). At telophase I, the homologs separate and form two clusters in WT (L); while some chromosomes more distant from the center are decondensed in p35S SDS-RNAi lines, resembling those in normal telophase I (arrowhead and arrow highlight that the chromosomes still stayed at the center and chromosome bridges, respectively, P); in p35S RCK-RNAi lines, most chromosomes are decondensed normally, but some are delayed (arrowheads, $\mathbf{X})$. Bar $=10 \mu \mathrm{m}$. 
A

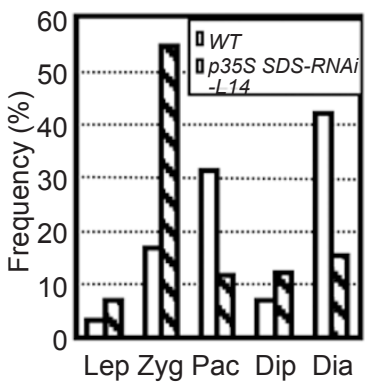

B

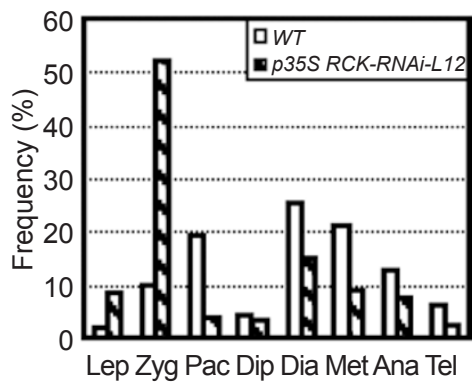

Figure 6 Distribution of meiosis I meiocytes of WT, p35S SDS$R N A i$ and $p 35 S$ RCK-RNAi lines. Chromosome spreads are examined as shown in Figure 5. Lep (leptotene), Zyg (zygotene), Pac (pachytene), Dip (diplotene), Dia (diakenesis of prophase I), Met (metaphase I), Ana (anaphase I) and Tel (telophase I). (A) Distribution of prophase I cells in WT (1 954 cells) and the p35S SDS-RNAi-L14 line (2 138 cells). (B) Distribution of meiosis I meiocytes of WT (3 232 cells) and p35S RCK-RNAi-L12 line (3 842 cells).

RNAi transgenic meiocytes indicates that some crossovers were formed. To examine this aspect of the meiotic phenotype, we counted the number of chiasmata. At early diakinesis, meiocytes of the $R C K$-deficient lines exhibited a reduction in chiasma number compared with wildtype meiocytes (Figure 7A). A sample of $140 \mathrm{p} 35 \mathrm{~S} R C K$ RNAi-L12 cells showed an average of 13.99 chiasmata per cell compared with an average of 23.90 chiasmata per cell in the wild-type meiocytes. Several univalents were observed in the $p 35 S R C K-R N A i-L 12$ cells (arrows in Figure 7A, right panel) in contrast to 12 bivalents during the comparable stage in the wild-type cells (Figure 7A, left panel). Among 140 p35S RCK-RNAi-L12 meiocytes at late prophase I to metaphase I, no meiocytes had 12 bivalents; 0.7, 0.7, 3.6, 7.9, 6.4, 8.6, 27.9, 19.3, 12.1, $7.1,5.7 \%$ were observed with $1,2,3,4,5,6,7,8,9,10$, 11 bivalents, respectively, with an average of 7.21 bivalents per cell (Figure 7C).

We analyzed the distribution of residual chiasmata present in p35S RCK-RNAi-L12 and compared them with that of the wild type (Figure 7B). The chiasma distribution for wild-type cells $(n=100)$ deviated significantly from the Poisson prediction (Figure 7B, top panel; $\chi_{(20)}^{2}$ $=364.65, P<0.005)$, whereas the chiasma distribution in $p 35 S R C K-R N A i-L 12$ cells $(n=140)$ was much closer to the predicted Poisson distribution (Figure $7 \mathrm{~B}$, bottom panel; $\left.\chi_{(17)}^{2}=32.73,0.01<P<0.025\right)$. The results demonstrate that $R C K$ deficiency likely causes severe defects in the interference-sensitive pathway of chiasma formation.
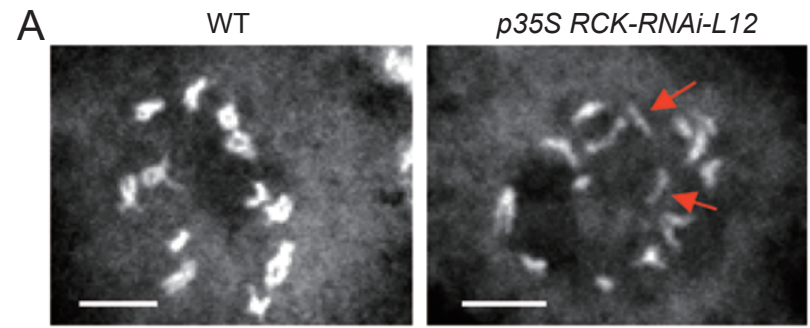

B
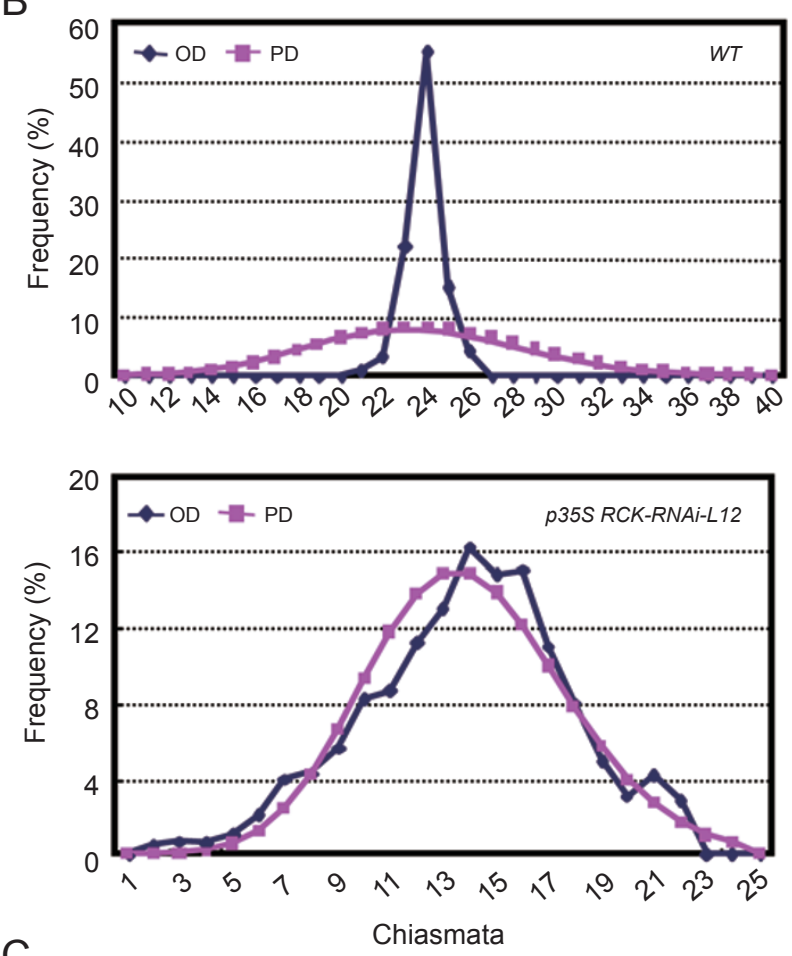

C

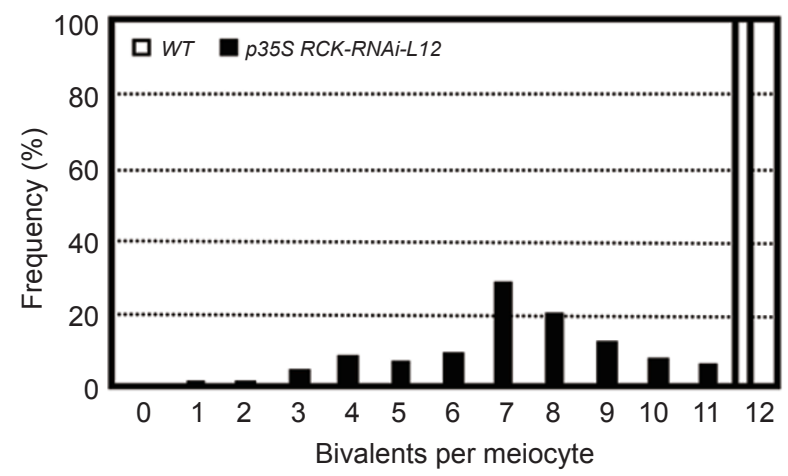

Figure 7 Distribution of chiasmata and bivalents of WT and p35S RCK-RNAi line. (A) Early diakinesis of WT (left) and p35S RCK-RNAi-L12 (right). Reduced chiamasta can be observed. Arrows highlight some univalents. Bar $=10 \mu \mathrm{m}$. (B) Frequency of chiasma number per meiocyte in WT (top panel, $\left.\chi_{(20)}^{2}=364.65, P<0.005, n=100\right)$ and p35S RCK-RNAi-L12 line (bottom panel, $\chi_{(17)}^{2}=32.73,0.01<P<0.025, n=140$ ). PD, predicted Poisson distribution; OD, observed distribution. (C) The frequency of bivalent number per meiocyte in WT and $p 35 S$ $R C K-R N A i-L 12$ lines. 


\section{Discussion}

\section{Evolution of SDS and RCK in flowering plants}

We have identified $S D S$ and $R C K$ homologs from several flowering plants, and phylogenetic analyses indicate that $S D S$ and its homologs form a well-supported clade separate from other cyclin genes, such as cyclin A and cyclin B genes. This phylogenetic relationship indicates that the $S D S$ homologs from flowering plants are likely true orthologs. For $R C K$, homologs were identified in several plants, as well as animals and fungi. The phylogenetic relationship of $R C K$ homologs is in agreement with the accepted relationship of the corresponding organisms, strongly suggesting that these genes are also orthologous. In addition, only one copy of $S D S$ and one copy of $R C K$ were found in Arabidopsis, poplar, and rice, plant species whose whole genome has been sequenced. It has been proposed that during the evolution of these species, there have been one or more genomewide duplication(s). Therefore, additional copies of $S D S$ must have been lost after the duplication(s), unlike the cyclin A and cyclin B genes, indicating that $S D S$ has a different pattern of evolution from those of other types of cyclin genes, which have expanded in flowering plants. The $R C K$ gene has also been maintained as single copy during eukaryotic evolution. The loss of duplicated SDS and $R C K$ genes in flowering plants suggests that additional copies are not beneficial, or even possibly deleterious to plants.

\section{Roles of SDS and RCK in rice male meiosis}

The observation that $S D S$ and $R C K$ are maintained as single copy genes in angiosperms suggests that they have conserved functions. Our studies using RNAi rice plants indicated that $S D S$ and $R C K$ are needed for normal rice meiosis and male fertility. In $S D S$-RNAi meiocytes, chromosome condensation during prophase I was normal, but there were many more zygotene cells and very few pachytene cells, suggesting that normal synapsis of homologs cannot be achieved under SDS deficiency, although a defect in pairing is also possible. Together with the observations of individual univalents at late prophase I, we conclude that the rice $S D S$ gene is required for homolog interaction and bivalent formation, similar to the Arabidopsis SDS function [20]. RCK-RNAi meiocytes also exhibited defects in meiotic crossover and bivalent formation. Compared to WT, the number of chiasmata in $R C K$-RNAi cells was dramatically reduced and the distribution of them was much closer to the Poisson distribution of random events, though the statistical analysis still showed a significant difference between the distribution of $R C K$-RNAi-L12 and Poisson distribution (Figure 7B, bottom panel). The relative few and close-to-randomly distributed crossovers in the RCK-RNAi plants suggest that rice, like Arabidopsis [3], also has two pathways for crossover formation: a major interference-sensitive $R C K$-dependent pathway and a minor pathway that is $R C K$-independent and interference insensitive. However, because the crossovers in the $R C K$-RNAi plants were not completely randomly distributed, the existence of a minor interference-insensitive pathway in rice, as that in Arabidopsis, needs further investigation. Phenotype of $R C K$-RNAi cells indicated that they were defective in the interference-sensitive pathway of meiotic recombination, similar to the phenotype of the Arabidopsis rck mutants [30]. Therefore, the functions of $S D S$ and $R C K$ are largely conserved in Arabidopsis and rice, which represent eudicots and monocots, respectively.

The relationship between recombination and synapsis is one of the most actively investigated aspects of meiosis. In yeast and Arabidopsis, synapsis and recombination are interdependent and closely coupled. In yeast, an important SC component HOP1, can preferentially bind to yeast DNA and promote crossover formation in meiotic recombination [52, 53]. Genetic studies strongly support the idea that SC is dependent on the initiation of recombination by DSBs. A mutation in the Arabidopsis ASY1 gene encoding an HOP1 homolog also causes a reduction in chiasmata number [54, 55]. In addition, Arabidopsis sds mutant also shows greatly reduced frequency of meiotic recombination [20], and some other mutants defective in meiotic recombination including atspo11-1, atrand51, atxrcc 3 and $r c k$ [8, 30, 56, 57] are unable to achieve normal SC formation, indicating that normal levels of recombination are needed for proper SC formation. Because SDS could potentially regulate gene expression indirectly, we tested whether rice $S D S$ can affect $R C K$ expression and found that the level of $R C K$ transcript

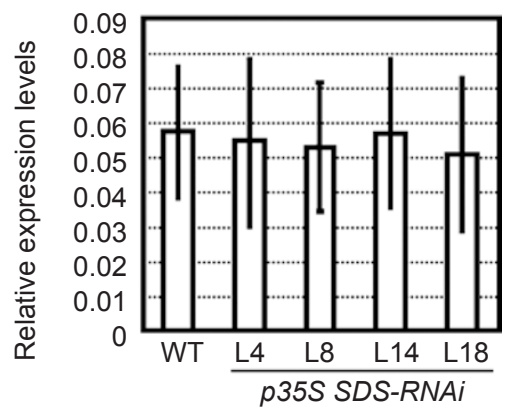

Figure 8 Evolutionary relationship between SDS and RCK. Real-time RCR analysis on the RCK transcripts in WT and p35S SDS-RNAi lines $(L 4,8,14,18)$. Total RNAs were extracted from flowers, and results are presented as an average of three independent experiments. 
was not influenced when $S D S$ expression was suppressed (Figure 8), suggesting that, though SDS and RCK function in two closely coupled processes of synapsis and recombination, they are not regulated by each other in rice.

\section{Conserved male meiosis in monocots and eudicots}

In addition to the conserved sequences and functions of rice $S D S$ and $R C K$ shown in this study, a small number of rice meiosis genes have been studied including PAIR2 [43], OsDMC1 [44], OsRad21-4 [45] and their functions in rice male meiosis are conserved compared to their correspondent homologous genes ASY1 [58], AtDMC1 [59, 60], SYN1 [61] in Arabidopsis. In Arabidopsis, the ASY1 protein is localized to the axial/lateral elements and an asyl mutant is asynaptic and unable to form SC in both male and female meiocytes, indicating its critical role in $\mathrm{SC}$ formation $[54,58,62]$. A mutation in PAIR2, the rice homolog of $A S Y 1$, causes defects in homolog alignment at pachytene and the formation of univalents instead of bivalents at diakinesis [63]. The PAIR2 proteins associate with axial elements (AEs) at leptotene and zygotene, and are removed from the AEs of arm regions when homologous chromosomes are synapsed [43]. The Arabidopsis dmcl mutant forms univalents instead of bivalents at meiotic prophase I and the recombination frequency is greatly reduced [59]. Similarly, knock down of rice $D M C 1$ leads to defects in bivalent formation and subsequent unequal chromosome segregation [44]. In Arabidopsis, sister chromatid cohesion is abnormal and chromosome condensation is also affected in the syn 1 mutant, and meiocytes of the mutant produce chromosomal fragments $[64,65]$. The male meiocytes in OSRAD21-4-deficient lines showed overcondensation of chromosomes, precocious segregation of homologs and chromosome fragmentation. Fluorescence in situ hybridization revealed that OsRAD21-4-deficient lines were defective in homologous pairing and cohesion at sister chromatid arms [45]. These results strongly support a conserved molecular mechanism for male meiosis in monocots and eudicots.

\section{Possible modified functions of SDS and RCK in Oryza sativa}

It is worth noting that some cytological characteristics in rice meiosis are different from that of Arabidopsis. The rice meiosis I is followed by the formation of a cell plate [41], instead of an organelle band, as observed in Arabidopsis, between the two daughter cells, indicating the differential aspects in meiosis of rice and Arabidopsis. Indeed, expression pattern analysis of the rice $S D S$ and $R C K$ genes demonstrated that although both of them were expressed preferentially in flowers, they were transcribed in other tissues as well. SDS was highly expressed in the shoot apex, suggesting that it might function in mitosis, which is different from the Arabidopsis $S D S$ gene that was only detectable in flowers containing meiotic cells and not in vegetative organs or other stages of reproductive structure [20]. Additionally, rice SDS expression in flowers was only detected in anther but not in pistils, which might be different from the Arabidopsis $S D S$, which functions in both female and male meiosis. Besides flowers, rice $R C K$ was also expressed in stems, leaves and seeds. Specifically, its expression was changed during floral development. In the flower before meiosis, $R C K$ expression was detected in both anthers and ovaries, while after meiosis stage, $R C K$ was mainly expressed in the ovaries. When the $p 35 S$ RCK-RNAi-L12 lines were pollinated with wild-type pollen, the mean seed setting rate was only $12.4 \%$, which is much lower when compared to the mean seed setting rate of wildtype lines $(53.9 \%)$, indicating that suppression of rice $R C K$ also reduced the female fertility. These results suggest that rice $R C K$ may have a modified function in male and female meiosis. In $p 35 S R C K-R N A i$ meiocytes, there were many zygotene cells and very few pachytene cells, similar to SDS-RNAi meiocytes; while the Arabidopsis $r c k$ mutants had an increased frequency of cells at the diakenesis stage [30]. Therefore, besides the conserved functions in chiasma and bivalent formation, rice $R C K$ may be required for other events of homolog interactions during prophase I such as synapsis. Further analysis is needed to determine the functional differences of $S D S$ and $R C K$ in rice.

\section{Materials and Methods}

\section{Plant materials}

Rice plants (Oryza sativa, japonica cultivar Zhonghua 11) were grown in a phytotron with a light/dark cycle of $12 \mathrm{~h}$ at $28{ }^{\circ} \mathrm{C} / 12 \mathrm{~h}$ at $22{ }^{\circ} \mathrm{C}$. Flowers in different stages were collected. Leaves and stems were collected from 6-week-old plants. Roots were collected from 1-week-old seedlings germinated in water.

\section{Isolation of rice SDS and RCK}

The cDNA sequences of $S D S$ and $R C K$ were amplified by RTPCR using the first-strand cDNA synthesized from rice flower RNAs as a template, with specific primers SDSF 1 (5'-GGC CCG TAG CAT CAC AAA TTC AC- $3^{\prime}$ ) and SDSR1 (5'-CGG CAT ATC ACC TGG GTT TGT TC-3'), and RCKF1 (5'-CGA GAC AGT AGC GCT AGC ACG AAC A-3') and RCKR1 (5'-TGC TGC ACA AGA TCG CCA CTG-3'). The amplified cDNA fragments were gel-purified, subcloned into the pGEM-T vector (Promega) and sequenced.

\section{Promoter-GUS fusion studies}

For further analysis of the expression pattern of $S D S$ and $R C K$, the 1.9-kb promoter region of rice $S D S$ was PCR amplified with 
primers SDSP1 (5'-TGG CGT TGG CTA AGA AGG TTG CTG$\left.3^{\prime}\right)$ and SDSP2 (5'-TGG CGT GCG GCG CTG TGT G-3'); the 2.5$\mathrm{kb}$ promoter region of $R C K$ was amplified with primers RCKP1 (5'-CGC GCA TGA ACA CGA TCC AAG-3') and RCKP2 (5'CGC GTG CGG TCC CTG CAG-3'), using rice genomic DNA as template. The resultant DNA fragments were subcloned into vector pCambia1300+pBI101 [66], resulting in the fusions with GUS reporter gene. The obtained constructs were introduced into Agrobacterium tumefaciens (strain EHA105) and transformed into the rice genome using immature embryo as materials [67]. Positive transgenic plants were obtained through selection by hygromycin resistance and PCR amplification. GUS activities were detected histochemically according to Jefferson et al. [68]. Photography was performed using a Nikon microscope with a digital camera.

\section{RNAi constructs}

A 478-bp intron amplified from rice genome with primers $\mathrm{InF}$ (5'-GTC TCTAGA GTA AGT TAC AAA CCT TTT TGT AC-3', $X b a$ I site underlined) and InR (5'-GCC GTCGAC CTG AAA ATC TCG AAA CAG CCG TGT C-3', SalI site underlined) was ligated into a pSK vector after digestion with $X b a \mathrm{I}$ and SalI, resulting in a vector pSK-Int. Then, two 535-bp fragments specific to SDS cDNA (nt 430-964) were amplified by PCR with primers SDSF2 (5'-AGCTCGAGT CCT ACC TCG CCT GCC CCG-3', XhoI site underlined) and SDSR2 (5'-TAGTCGACG CCC TAC CCC ATG AAC ACG GTC-3', SalI site underlined), and primers SDSF3 (5'-TAGGATCCT CCT ACC TCG CCT GCC CCG-3', BamHI site underlined) and SDSR3 (5'-TATCTAGAG CCC TAC CCC ATG AAC ACG GTC-3', XbaI site underlined). After digestion with corresponding restriction enzymes, the two fragments were subcloned into vector pSK-Int to generate the construct pSK-SDSi. Finally, the fragment in pSK-Int containing the 478-bp intron flanked with two 535-bp opposite fragments was digested with XhoI and BamHI and subcloned into pCAMBIA1300S, resulting the RNAi construct, p35S SDS-RNAi.

In a similar strategy, two 514-bp fragments specific to RCK cDNA (nt 2 409-2 922) were amplified by PCR with primers RCKF2 (5'-AGCTCGAGT GCT CCT TGC GAA ATG CTT ACA C-3', Xhol site underlined) and RCKR2 (5'-TAGTCGACT CCC TGC TGA CCA CAT GCT TCT TG-3', SalI site underlined), and primers RCKF3 (5'-TAGAGCTC T GCT CCT TGC GAA ATG CTT ACA C-3', SacI site underlined) and RCKR3 (5'-TATCTAGAT CCC TGC TGA CCA CAT GCT TCT TG-3', XbaI site underlined). The obtained DNA fragments were used to generate the RNAi vector of RCK, p35S RCK-RNAi. The resultant constructs were transformed into rice genome by A. tumefaciens-mediated transformation.

\section{Phenotypic analysis}

Fluorescein diacetate and $\mathrm{I}_{2}$-KI were used to determine the viability of pollen grains. Mature flowers before opening were harvested and picked anthers were crushed into a fine powder and stained with $10 \mu \mathrm{l} \mathrm{I}_{2}$-KI solution ( $1 \mathrm{~g} \mathrm{KI}$ and $0.5 \mathrm{~g} \mathrm{I}_{2}$ dissolved in $100 \mathrm{ml}$ distilled water) for a couple of minutes [69] and observed under a microscope (Leica DMR, Germany). Pollen grains that were round in shape and stained dark blue-black were regarded as viable or living pollen. The sterile or dead pollens were stained brown.

Anthers were treated with FDA solution (10 mg FDA dissolved in $1 \mathrm{ml}$ acetone as stock, diluted with 7\% sucrose to a concentration of $100 \mu \mathrm{g} / \mathrm{ml}$ for use) for $5 \mathrm{~min}$, and then photographed under ultraviolet light (Leica DMR, Germany) [70]. Pollen grains emitting green fluorescence were regarded as viable pollen. Pollen number per flower was counted with a hematocyte arithmometer, and the data is estimated from 30 independent flowers for each line [45].

Chromosome spreading and 4,6-Diamidino-2-phenylindole (DAPI) staining were performed according to Ross et al. [71] with some modifications. Inflorescences collected from rice shoots were fixed in the Carnoy's fixative (methanol: glacial acetic acid $=3: 1$, $\mathrm{v}: \mathrm{v})$ at room temperature at least for $4 \mathrm{~h}$. Then the fixed flowers were washed with $10 \mathrm{mM}$ citrate buffer, $\mathrm{pH} 4.5$, and digested with $0.3 \%$ cytohelicase, $0.3 \%$ cellulose and $0.3 \%$ pectolyase in citrated buffer at $37{ }^{\circ} \mathrm{C}$ for $30 \mathrm{~min}$. The digested anthers were washed with citrate buffer and placed in a small drop of $60 \%$ acetic acid on a slide, then squashed with a cover slip to release microspore mother cell. After the samples were dried at room temperature, $5 \mu 1$ DAPI solution $(1 \mu \mathrm{g} / \mathrm{ml}$ DAPI, 50\% glycerol, $10 \mathrm{mM}$ citrate buffer, $\mathrm{pH}$ 4.5) was added onto the slide and then observed under a confocal microscope (Carl Zeiss LSM510 META, Germany). Numbers of the chiasma and bivalent/univalent were counted according to the methods previously described $[20,30,72]$.

\section{Quantitative real-time RT-qPCR analysis}

Total RNA was extracted using TRizol reagent (Invitrogen) and reverse-transcripted according to the manufacturer's instructions (SuperScript preamplification system; Promega). The primers used for SDS were SDSF4 (5'-AGT GAG GTC GTC GCC ATG GAG TG-3') and SDSR4 (5'-GGA GGG CCA GAA GGA GAG ATG TTT G-3'). The primers used for $R C K$ were RCKF4 (5'-AGC ATG TGG TCA GCA GGG AAG ATT T-3') and RCKR4 (5'-GGG CCT CCA GTG AAG CTA AGT TGT TG-3'). Rice ACTIN1 was used as an internal control with primers 5'-GAA CTG GTA TGG TCA AGG CTG-3' (forward) and 5'-ACA CGG AGC TCG TTG TAG AAG-3' (reverse).

RT-qPCR was performed on a RotorGene 3000 system (Corbett Research) using a SYBR green detection protocol according to the manufacturer's instructions (SYBR Premix Ex Taq System; TOYOBO). A linear standard curve and threshold cycle number versus log (designated transcript level) were constructed using a series of dilutions of each PCR product; and the levels of the transcript in all unknown samples were determined according to the standard curve. The ACTIN1 was used for normalizing cDNA concentration variations. The experiments were repeated thrice.

\section{Phylogenetic analysis}

Homolog sequences in P. trichocarpa, Selaginella moellendorffii and Physcomitrella patens were obtained at JGI website (http://genome.jgi-psf.org), other homologous sequences were obtained from PsiBlast searches at the National center for Biotechnology Information (http://blast.ncbi.nlm.nih.gov/Blast.cgi). The conserved domains of proteins were analyzed at the website http://www.ncbi.nlm.nih.gov/Structure/cdd/wrpsb.cgi. Since all the SDS homologs had both the cyclin_N and cylcin_C domains and all the RCK homologs had the conserved domains including DEXDc, HELICc and SEC63, we used the conserved domains for phylogenetic analyses. Sequence alignments were generated with CLUSTALX 1.83 [73], and the alignments between $A$. thaliana, $P$. 
trichocarpa and $V$. vinifera SDS were adjusted before constructing the tree. Neighbor-joining analyses were performed using MEGA3 [74] with the pairwise deletion option, Poisson correction set for distance model and 1000 bootstrap replicates selected. Maximum likelihood analyses were performed on the PhyML online (http:// atgc.lirmm.fr/phyml/) with 100 bootstrap replicates selected [75, 76].

\section{Acknowledgments}

This work was supported by the State Key Project of Basic Research (2005CB120803), the Chinese Academy of Sciences (KSCX2-YW-N-058) and Reproductive Development Project from the Shanghai Institutes for Biological Sciences. We thank Zhu-Yun Deng at the Institute of Botany, Chinese Academy of Sciences, for technical advice in chromosome spreading and DAPI staining, and Xinwei Han in the Intercollege Graduate Program in Genetics and Department of Biology, the Pennsylvania State University, USA, for advice on phylogenetic analysis.

\section{References}

1 Armstrong SJ, Jones GH. Meiotic cytology and chromosome behaviour in wild-type Arabidopsis thaliana. J Exp Bot 2003; 54:1-10.

2 Dawe RK. Meiotic chromosome organization and segregation in plants. Annu Rev Plant Physiol Plant Mol Biol 1998; 49:371-195

3 Ma H. A molecular portrait of Arabidopsis meiosis. In: CR Somerville, EM Meyerowitz, J Dangl, M Stitt, eds. The Arabidopsis Book. Rockville, MD, USA: American Society of Plant Biologists, 2006. doi/10.1199/tab.0009, http://www. aspb.org/publications/arabidopsis/.

4 Stack SM, Anderson LK. A model for chromosome structure during the mitotic and meiotic cell cycles. Chromosome Res 2001; 9:175-198.

5 Zickler D, Kleckner N. Meiotic chromosomes: integrating structure and function. Annu Rev Genet 1999; 33:603-754.

6 Zickler D, Kleckner N. The leptotene-zygotene transition of meiosis. Annu Rev Genet 1998; 32:619-697.

7 Cervantes MD, Farah JA, Smith GR. Meiotic DNA breaks associated with recombination in S. pombe. Mol Cell 2000; 5:883-888.

8 Grelon M, Vezon D, Gendrot G, Pelletier G. AtSPO11-1 is necessary for efficient meiotic recombination in plants. EMBO J 2001; 20:589-600.

9 Li W, Chen C, Markmann-Mulisch U, et al. The Arabidopsis AtRAD51 gene is dispensable for vegetative development but required for meiosis. Proc Natl Acad Sci USA 2004; 101:10596-10601.

10 Romanienko PJ, Camerini-Otero RD. The mouse Spoll gene is required for meiotic chromosome synapsis. Mol Cell 2000; 6:975-987.

11 Zenvirth D, Simchen G. Meiotic double-strand breaks in Schizosaccharomyces pombe. Curr Genet 2000; 38:33-38.

12 Dernburg AF, McDonald K, Moulder G, Barstead R, Dresser M, Villeneuve AM. Meiotic recombination in C. elegans initiates by a conserved mechanism and is dispensable for homologous chromosome synapsis. Cell 1998; 94:387-398.
13 Mckim KS, Green-Marroquin BL, Sekelsky JJ, et al. Meiotic synapsis in the absence of recombination. Science 1998; 297:876-878.

14 Andrews B, Measday V. The cyclin family of budding yeast: abundant use of a good idea. Trends Genet 1998; 14:66-72.

15 Gitig DM, Kof A. CDK pathway: cyclin-dependent kinases and cyclin-dependent kinase inhibitors. Methods Mol Biol 2000; 142:109-123.

16 Murray AW. Cyclin-dependent kinases: regulators of the cell cycle and more. Chem Biol 1994; 1:191-195.

17 Dahamann C, Futcher B. Specialization of B-type cyclins for mitosis or meiosis in S. cerevisiae. Genetics 1995; 140:957963.

18 Grandin N, Reed SI. Differential function and expression of Saccharomyces cerevisiae B-type cyclins in mitosis and meiosis. Mol Cell Biol 1993; 13:2113-2125.

19 Liu D, Matzuk MM, Sung W K, Guo Q, Wang P, Wolgemuth DJ. Cyclin A1 is required for meiosis in the male mouse. $\mathrm{Na}$ ture Genet 1998; 20:377-380.

20 Azumi Y, Liu D, Li W, Wang G, Hu Y, Ma H. Homolog interaction during meiotic prophase I in Arabidopsis requires the SOLO DANCERS gene encoding a novel cyclin-like protein. EMBO J 2002; 21:3081-3095.

21 Szostak JW, Orr-Weaver TL, Rothstein RJ, Stahl FW. The double-strand-break repair model for recombination. Cell 1983; 33:25-35.

22 Maloisel L, Bhargava J, Roeder GS. A role for DNA polymerase delta in gene conversion and crossing over during meiosis in Saccharomyces cerevisiae. Genetics 2004; 167:1133-1142.

23 Keeney S. Mechanism and control of meiotic recombination initiation. Curr Top Dev Biol 2001; 52:1-53.

24 Lichten M. Meiotic recombination: breaking the genome to save it. Curr Biol 2001; 11:R253-R256.

25 Hollingsworth NM, Ponte L, Halsey C. MSH5, a novel MutS homolog, facilitates meiotic reciprocal recombination between homologs in Saccharomyces cerevisiae but not mismatch repair. Genes Dev 1995; 9:1728-1739.

26 Nakagawa T, Ogawa H. The Saccharomyces cerevisiae MER3 gene, encoding a novel helicase-like protein, is required for crossover control in meiosis. EMBO J 1999; 18:5714-5723.

27 Nakagawa T, Kolodner RD. Saccharomyces cerevisiae Mer3 is a DNA helicase involved in meiotic crossing over. Mol Cell Biol 2002; 22:3281-3291.

28 Novak JE, Ross-Macdonald PB, Roeder GS. The budding yeast Msh4 protein functions in chromosome synapsis and the regulation of crossover distribution. Genetics 2001; 158:10131025.

29 Mazina OM, Mazin AV, Nakagawa T, Kolodner RD, Kowalczykowski SC. Saccharomyces cerevisiae Mer3 helicase stimulates 3'-5' heteroduplex extension by Rad51; implications for crossover control in meiotic recombination. Cell 2004; 117:47-56

30 Chen CB, Zhang W, Timofejeva L, Gerardin Y, Ma H. The Arabidopsis ROCK-N-ROLLERS gene encodes a homolog of the yeast ATP-dependent DNA helicase MER3 and is required for normal meiotic crossover formation. Plant J 2005; 43:321-334.

31 Mercier R, Jolivet S, Vezon D, et al. Two meiotic crossover 
classes cohabit in Arabidopsis: one is dependent on MER3, whereas the other one is not. Curr Biol 2005; 15:692-701.

32 Borner GV, Kleckner N, Hunter N. Crossover/non-crossover differentiation, synaptonemal complex formation, and regulatory surveillance at the leptotene/zygotene transition of meiosis. Cell 2004; 177:29-45.

33 Copenhaver GP, Houseworth EA, Stahl FW. Crossover interference in Arabidopsis. Genetics 2002; 160:1631-1639.

34 Egel R. The synaptonemal complex and the distribution of meiotic recombination events. Trends Genet 1995; 11:206208.

35 Moens PB. Ultrastructural studies of chiasma distribution. Annu Rev Genet 1978; 12:433-450.

36 Whitehouse HLK. Towards an Understanding of the Mechanism of Heredity. New York: St Martin's Press, 1973.

37 Page SL, Hawley RS. The genetics and molecular biology of the synaptonemal complex. Annu Rev Cell Dev Biol 2004; 20:525-558.

38 De los Santos T, Hunter N, Lee C, Larkin B, Loidl J, Hollingsworth NM. The Mus81/Mms4 endonuclease acts independently of double-Holliday junction resolution to promote a distinct subset of crossovers during meiosis in budding yeast. Genetics 2003; 164:81-94.

39 Stahl FW, Foss HM, Young LS, Borts RH, Abdullah MF, Copenhaver GP. Does crossover interference count in Saccharomyces cerevisiae? Genetics 2004; 168:35-48.

40 Wijeratne AJ, Ma H. Genetic analyses of meiotic recombination in Arabidopsis. J Integr Plant Biol 2007; 49:1199-1207.

41 Chen $\mathrm{C}, \mathrm{Xu} \mathrm{Y,} \mathrm{Ma} \mathrm{H,} \mathrm{Chong} \mathrm{K.} \mathrm{Cell} \mathrm{biological} \mathrm{characteriza-}$ tion of male meiosis and pollen development in rice. J Integr Plant Biol 2005; 47:734-744.

42 Nonomura K, Nakano M, Fukuda T, et al. The novel gene HOMOLOGOUS PAIRING ABERRATION IN RICE MEIO$S I S 1$ of rice encodes a putative coiled-coil protein required for homologous chromosome pairing in meiosis. Plant Cell 2004; 16:1008-1020.

43 Nonomura K, Nakano M, Murata K, et al. An insertional mutation in the rice PAIR2 gene, the ortholog of Arabidopsis $A S Y 1$, results in a defect in homologous chromosome pairing during meiosis. Mol Genet Genomic 2004; 271:121-129.

44 Deng ZY, Wang T. OsDMC1 is required for homologous pairing in Oryza sativa. Plant Mol Biol 2007; 65:31-42.

45 Zhang LR, Tao JY, Wang SX, Chong K, Wang T. The Rice OsRad21-4, an orthologue of yeast Rec8 protein, is required for efficient meiosis. Plant Mol Biol 2006; 60:533-554.

46 Tao JY, Zhang LR, Chong K, Wang T. OsRAD21-3, an orthologue of yeast RAD21, is required for pollen development in Oryza sativa. Plant J 2007; 51:919-930.

47 Nakagawa T, Flores-Rozas H, Kolodner RD. The MER3 helicase involved in meiotic crossing over is stimulated by singlestranded DNA-binding proteins and unwinds DNA in the $3^{\prime}-5^{\prime}$ direction. J Biol Chem 2001; 276:31487-31493.

48 Shibata A, Nakagawa N, Sugahara M, et al. Crystallization and preliminary X-ray diffraction studies of a DNA repair enzyme, UvrB, from Thermus thermophilus HB8. Acta Crystalogr D Biol Crystallogr 1999; 55:704-705.

$49 \mathrm{Ng}$ DT, Brown JD, Walter P. Signal sequences specify the targeting route to the endoplasmic reticulum membrane. $J$ Cell Biol 1996; 134:269-278.
50 Noble SM, Guthrie C. Identification of novel genes required for yeast pre-mRNA splicing by means of cold-sensitive mutations. Genetics 1996; 143:67-80.

51 Wang GF, Kong HZ, Sun YJ, et al. Genome-wide analysis of the cyclin family in Arabidopsis and comparative phylogenetic analysis of plant cyclin-like proteins. Plant Physiol 2004; 135:1084-1099.

52 Alche JD, Paul E, Dickinson H. Heterologously expressed polypeptide from the yeast meiotic gene $H O P 1$ binds preferentially to yeast DNA. Protein Expr Purif 1999; 16:251-260.

53 Muniyappa K, Anuradha S, Byers B. Yeast meiosis-specific protein Hop1 binds to G4 DNA and promotes its formation. Mol Cell Biol 2000; 20:1361-1369.

54 Caryl AP, Armstrong SJ, Jones GH, Franklin FC. A homologue of the yeast $H O P 1$ gene is inactivated in the Arabidopsis meiotic mutant asy1. Chromosoma 2000; 109:62-71.

55 Sanchez-Moran E, Armstrong SJ, Santos JL, Franklin FC, Jones GH. Chiasma formation in Arabidopsis thaliana accession Wassileskija and in two meiotic mutants. Chromosome Res 2001; 9:121-128.

56 Bleuyard JY, White CI. The Arabidopsis homologue of Xrcc3 plays an essential role in meiosis. EMBO J 2004; 23:439-449.

57 Higgins JD, Armstrong SJ, Franklin FC, Jones GH. The Arabidopsis MutS homolog AtMSH4 functions at an early step in recombination: evidence for two classes of recombination in Arabidopsis. Genes Dev 2004; 18:2557-2570.

58 Armstrong SJ, Caryl AP, Jones GH, Franklin FC. Asy1, a protein required for meiotic chromosome synapsis, localizes to axis-associated chromatin in Arabidopsis and Brassica. J Cell Sci 2002; 115:3645-3655.

59 Couteau F, Belzile F, Horlow C, Grandjean O, Vezon D, Doutriaux MP. Random chromosome segregation without meiotic arrest in both male and female meiocytes of a $d m c 1$ mutant of Arabidopsis. Plant Cell 1999; 11:1623-1634.

60 Doutriaux MP, Couteau F, Bergounioux C, White C. Isolation and characterization of the RAD51 and DMC1 homologs from Arabidopsis thaliana. Mol Gen Genet 1998; 257:283291.

61 Cai X, Dong F, Edelmann RE, Makaroff CA. The Arabidopsis SYN1 cohesin protein is required for sister chromatid arm cohesion and homologous chromosome pairing. J Cell Sci 2003; 116:2999-3007.

62 Ross KJ, Fransz P, Armstrong SJ, Vizir I, Mulligan B. Cytological characterization of four meiotic mutants of Arabidopsis isolated from T-DNA-transformed lines. Chromosome Res 1997; 5:551-559.

63 Nonomura KI, Nakano M, Murata K, et al. An insertional mutation in the rice PAIR2 gene, the ortholog of Arabidopsis $A S Y 1$, results in a defect in homologous chromosome pairing during meiosis. Mol Genet Genomics 2004; 271:121-129.

64 Bai XF, Peirson BN, Dong FG, Xue C, Makaroff CA. Isolation and characterization of SYN1, a RAD21 like gene essential for meiosis in Arabidopsis. Plant Cell 1999; 11:417-430.

65 Peirson BN, Bowling SE, Makaroff CA. A defect in synapsis causes male sterility in a T-DNA-tagged Arabidopsis thaliana mutant. Plant J 1997; 11:659-669.

66 Liu W, Xu ZH, Luo D, Xue HW. Roles of OsCK11, a rice casein kinase I, in root development and plant hormone sensitivity. Plant J 2003; 36:189-202. 
67 Hiei Y, Ohta S, Komari T, Kumashiro T. Efficient transformation of rice (Oryza sativa L.) mediated by Agrobacterium and sequence analysis of the boundaries of the T-DNA. Plant $J$ 1994; 6:271-282.

68 Jefferson RA, Kavanagh TA, Bevan MW. GUS fusions: $\beta$-Glucuronidase as a sensitive and versatile gene fusion marker in higher plants. EMBO J 1987; 6:3901-3907.

69 Bolat I, Pirlak L. An investigation on pollen viability, germination and tube growth in some stone fruits. Turkish J Agric Forestry 1999; 23:383-388.

70 Falque M, Kodia AA, Sounigo O, Eskes AB, Charrier A. Gamma-irradiation of cacao (Theobroma cacao L.) pollen: effect on pollen grain viability, germination and mitosis and on fruit set. Euphytica 1992; 64:167-172.

71 Ross KJ, Fransz P, Jones GH. A light microscopic atlas of meiosis in Arabidopsis thaliana. Chromosome Res 1996;
4:507-516.

72 Sanchez-Moran E, Armstrong SJ, Santos JL, et al. Chiasma formation in Arabidopsis thaliana accession Wassileskija and in two meiotic mutants. Chromosome Res 2001; 9:121-128.

73 Henikoff S, Henikoff JG. Amino acid substitution matrices from protein blocks. Proc Natl Acad Sci USA 1992; 89:1091510919.

74 Kumar S, Tamura K, Nei M. MEGA 3, Integrated software for molecular evolutionary genetics analysis and sequence alignment. Brief Bioinform 2004; 5:150-163.

75 Guindon S, Gascuel O. A simple, fast, and accurate algorithm to estimate large phylogenies by maximum likelihood. Syst Biol 2003; 52:696-704.

76 Guindon S, Lethiec F, Duroux P, Gascuel O. PHYML Onlinea web server for fast maximum likelihood-based phylogenetic inference. Nucleic Acids Res 2005; 33:557-559. 